Paper

\title{
Bending angles of a broken line causing bifurcations and chaos
}

\author{
Yuu Miino $^{1 a)}$, Tetsushi Ueta ${ }^{2}$, and Hiroshi Kawakami ${ }^{2}$ \\ ${ }^{1}$ Tokyo University of Technology, \\ 1404-1, Katakuramachi, Hachioji, Tokyo 192-0982, Japan \\ ${ }^{2}$ Tokushima University, \\ 2-1, Minami-Josanjima-cho, Tokushima 770-8506, Japan \\ a) miinoy@stf.teu.ac.jp
}

Received August 6, 2019; Revised January 9, 2020; Published July 1, 2020

\begin{abstract}
We replace the cubic characteristics in the Duffing equation by two line segments connected at a point and investigate how an angle of that broken line conducts bifurcations to periodic orbits. Firstly we discuss differences in periodic orbits between the Duffing equation and a forced planar system including the broken line. In the latter system, a grazing bifurcation split the parameter space into the linear and nonlinear response domains. Also, we show that bifurcations of non-resonant periodic orbits appeared in the former system are suppressed in the latter system. Secondly, we obtain bifurcation diagrams by changing a slant parameter of the broken line. We also find the parameter set that a homoclinic bifurcation arises and the corresponding horseshoe map. It is clarified that a grazing bifurcation and tangent bifurcations form boundaries between linear and nonlinear responses. Finally, we explore the piecewise linear functions that show the minimum bending angles exhibiting bifurcation and chaos.
\end{abstract}

Key Words: local and global bifurcation analysis, piecewise linear dynamical system, hybrid dynamical system, Duffing equation

\section{Introduction}

There is plenty of studies on nonlinear differential equations derived from mechanics $[1,2]$, electronics $[3,4]$, biology $[5,6]$, and so on. These systems often exhibit a rich variety of responses, i.e., stable and unstable periodic orbits, and chaos. Among them, bifurcations play a very important role, e.g., a tangent bifurcation generates or extinguishes a couple of a stable fixed point and a saddle point, and a period-doubling bifurcation unstabilizes a stable fixed point and generates 2-periodic points. The bifurcations caused in individual systems have been well studied [7], however, as far as authors investigated, few discussions are focusing on the nonlinearity to exhibit the nonlinear phenomena.

Recently, on the other hand, hybrid dynamical systems (HDSs) are actively studied. HDSs contain a useful scheme to construct the realistic systems: neural dynamics [5], conflict systems of objects [8], circuits including a diode [9] and so on [10,11]. Kousaka et al. [12] have suggested the method to solve the local bifurcation problem of an HDS and Banerjee [13] has indicated that there exist some conspicuous phenomena in the systems: a grazing bifurcation and so on. We have also studied an 
HDS whose conditions depending on the states or the past time [14]. Even if a system includes very complex characteristics, PWL functions can faithfully approximate them. Moreover, in some specified cases, it might be possible to obtain an analytical solution. Tanaka et al. [9] had expressed the diode characteristics by the PWL function. Their circuit is a driven RL-diode circuit, and their numerical experiments display the bifurcations and chaos in the time-discrete system approximated from the circuit. On the other hand, Shaw et al. [15] has studied the local bifurcations and chaotic motions in the system whose structure is almost the same as the driven RL-diode circuit. They have used the normalized circuit equation as it is and given the bifurcation sets and the invariant curves of some specified solutions by the analytical method. These methods are available for some specified solutions: a 1-periodic orbit, or a 2-periodic orbit, and so on.

We can flexibly construct PWL dynamical systems by using HDSs and can solve their bifurcation problem of the system by both analytical and numerical methods. Then, our interest is that the minimum bending angle of the PWL function required to exhibit the nonlinear phenomena. There is no doubt the bending angle is one index of the nonlinearity. Thus, in this study, we explore how does a broken line, which is the simplest PWL function, brings bifurcations and chaos to a forced planar system. Firstly, we explain a simple dynamical system including the PWL function, which is a dual circuit of the driven RL circuit (Sec. 2.1). Assume that the nonlinear term in the system is only this PWL function and the slope of this function is a free parameter. Basic definitions for analysis of the dynamical system are also defined at the same time. Since the system can become completely linear with a particular parameter setting, we mention the condition of this parameter from a mathematical derivation (Sec. 2.2). Secondly, we describe the method for the bifurcation analysis (Sec. 3). We define the proper Poincaré map of the system and the variational equation; then we obtain the characteristic multipliers of the periodic orbit (Sec. 3.1). We show the conditions for the local bifurcation phenomena observed in the system. To discuss global bifurcation phenomena, we introduce the stable and the unstable manifolds of a saddle and describe the method to calculate the parameter set causing homoclinic bifurcation phenomena (Sec. 3.2). This scheme is mainly based on the method proposed by Yoshinaga et al. [16]. Since the model equation of an HDS is not smooth, we should take a special scheme [14] to calculate the Jacobian matrix of a Poincaré map. On the other hand, HDSs often include a threshold which sometimes influences the stability of the periodic orbit. We call this change of stability a grazing bifurcation phenomenon and derive the condition for this bifurcation. As a result of the analysis, we indicate the existence of the horseshoe map and plot the bifurcation sets in the two-dimensional parameter space (Sec. 4). We discuss differences and similarities of periodic orbits between the Duffing equation and our system (Sec. 4.2). We show some periodic orbits in the state space and concretely explain bifurcations and nonlinear resonances as illustrations. Assuming that the slope of the PWL function is a variable parameter, we explore the boundary of the nonlinear and the linear systems. Focusing on global bifurcation phenomena, we show the manifolds of a saddle before and after a homoclinic bifurcation (Sec. 4.3). We also confirm the existence of the horseshoe map [17] by setting a particular parameter. Finally, we summarize this study (Sec. 5).

\section{System description}

\subsection{Forced planar system including a piecewise linear function}

Let us introduce a continuous time, non-autonomous, dynamical system defined by:

$$
\frac{d x}{d t}=y, \quad \frac{d y}{d t}=-k y-f(x)+B_{0}+B \cos t,
$$

where $t$ is the time, $x$ and $y$ are the state variables, and $k \geq 0, B_{0} \geq 0$ and $B \geq 0$ are the parameters. We also use the vector notation for these state variables: $\boldsymbol{x}=(x, y)$. If the function $f$ of $x$ is a cubic function, as shown in Fig. 1(a), the system (1) is generally called the Duffing equation, which has already been studied by Kawakami et al. [18]. We make the system (1) piecewise linear by defining the function $f$ of $x$ as the following function: 
(a)

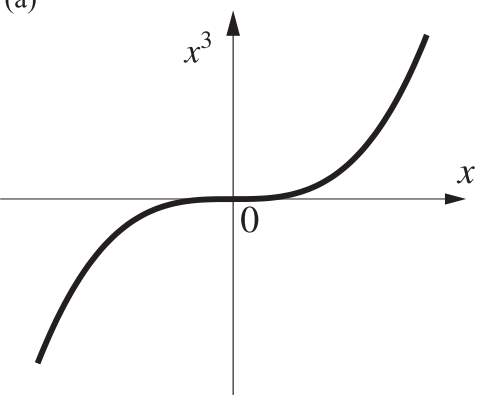

(b)

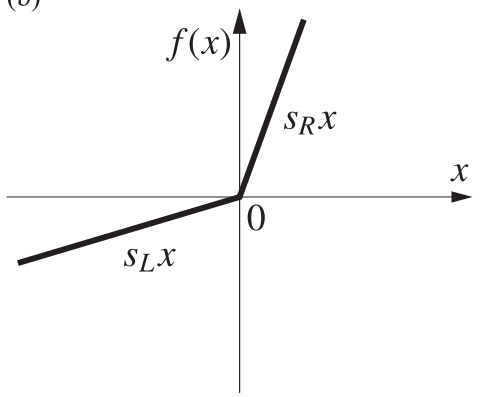

(c)

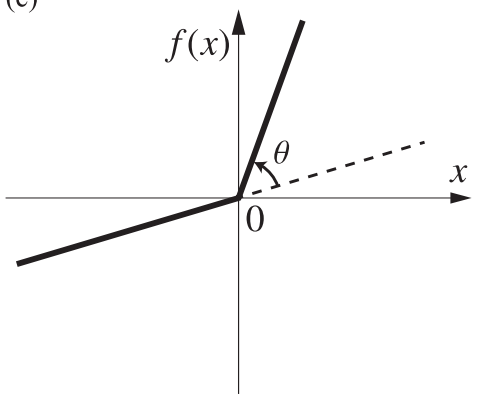

Fig. 1. (a) The cubic function in the Duffing equation, (b) the characteristic of $f(x)$ in the system (1), and (c) the bending angle $\theta$ of $f(x)$.

$$
f(x)= \begin{cases}s_{L} x & \text { if } \quad x<0 \\ s_{R} x & \text { if } \quad x \geq 0\end{cases}
$$

where $s_{L} \geq 0$ and $s_{R} \geq 0$ are parameters controlling the slope of the linear functions included in $f(x)$. Then the definition of the bending angle $\theta$ of $f(x)$ is

$$
\theta=\tan ^{-1} s_{R}-\tan ^{-1} s_{L}
$$

which measures how $f(x)$ is bending comparing with a linear function. Figures $1(\mathrm{~b})$ and (c) show the characteristic and $\theta$ of $f(x)$. We call the line $x=0$ in the state space the border of the system (1) because it divides the state space into two subspaces; we hereby define the border as a function of the state vector: $q(\boldsymbol{x})=x$. On these two subspaces, different linear dynamical systems determine individual vector fields. Notice that $f(x)$ is the only nonlinear term in the system (1). In Ref. of Tanaka et al. [9], they have proposed an electric circuit holding the similar system as the system (1). On the other hand, Zhao et al. [19] have also suggested the result of stability analysis for the similar system.

An orbit $\boldsymbol{x}(t) \in \boldsymbol{R}^{2}$ is the solution of Eq. (1) together with an initial value $\boldsymbol{x}_{0}=\left(x_{0}, y_{0}\right) \in \boldsymbol{R}^{2}$. We define $\boldsymbol{x}(t)$ by a function of time $t$ and $\boldsymbol{x}_{0}$ :

$$
\boldsymbol{x}(t)=\boldsymbol{\varphi}\left(t, \boldsymbol{x}_{0}\right), \quad \boldsymbol{x}(0)=\boldsymbol{\varphi}\left(0, \boldsymbol{x}_{0}\right)=\boldsymbol{x}_{0}
$$

We obtain the orbits by solving the Runge-Kutta method. An orbit $x(t)$ is periodic if there exists a constant $L>0$ such that

$$
\boldsymbol{x}(t+L)=\boldsymbol{x}(t), \quad \forall t \in \boldsymbol{R} .
$$

The period of this orbit is the smallest value for which (5) holds.

\subsection{Linear periodic orbit}

Let us define the domains $M_{-}=\left\{(x, y) \in \boldsymbol{R}^{2} \mid x<0\right\}$ and $M_{+}=\left\{(x, y) \in \boldsymbol{R}^{2} \mid x \geq 0\right\}$. We find a linear periodic orbit confined within $M_{+}$under the condition:

$$
\frac{B_{0}}{s_{R}}-\frac{B}{\sqrt{k^{2}+\left(1-s_{R}\right)^{2}}} \geq 0 .
$$

With equality, the periodic orbit is tangent to the line $x=0$, and the grazing bifurcation occurs. Similarly, we also find another linear periodic orbit within $M_{-}$, however, the orbit is not appear with our parameters.

\section{Method of analysis}

In this section, we describe the cause of emerging bifurcation phenomena exhibited in the system (1) and summarize the method to obtain the bifurcation parameter. 


\subsection{Local bifurcations}

Discussing the asymptotic stability of the periodic orbit, we generally introduce the Poincaré map [20] of the orbit, which is given as the discrete map as follows:

$$
\begin{aligned}
T: & \boldsymbol{R}^{2} \rightarrow \boldsymbol{R}^{2} ; \\
& \boldsymbol{x}_{0} \mapsto T\left(\boldsymbol{x}_{0}\right)=\boldsymbol{\varphi}\left(2 \pi, \boldsymbol{x}_{0}\right) .
\end{aligned}
$$

In the field of discrete-time dynamical systems, the point $\boldsymbol{p} \in \boldsymbol{R}^{2}$ is called a fixed point of the map $T$ if

$$
T(\boldsymbol{p})=\boldsymbol{p} .
$$

Similarly, the point $\boldsymbol{p}$ is called an $\ell$-periodic point of the map $T$ if

$$
T^{\ell}(\boldsymbol{p})=\boldsymbol{p},
$$

but $T^{k}(\boldsymbol{p}) \neq \boldsymbol{p}$ for $k=1, \ldots, l-1$ where $l=2,3, \ldots$. These periodic points of $T$ exactly correspond to the periodic orbit of the system (1).

The variational equation from the fixed point $\boldsymbol{p}$ with respect to the Poincaré map $T$ is given as follows:

$$
\boldsymbol{u}_{n+1}=\left.\frac{\partial T}{\partial \boldsymbol{x}_{0}}\right|_{\boldsymbol{x}_{0}=\boldsymbol{p}} \boldsymbol{u}_{n}, \quad n=0,1, \ldots
$$

where $\boldsymbol{u}_{n}$ is the difference between $\boldsymbol{p}$ and $T^{n}\left(\boldsymbol{p}+\boldsymbol{u}_{0}\right)$, and $\boldsymbol{u}_{0} \in \boldsymbol{R}^{2}$ is an arbitrary vector having a small norm. The multipliers $\mu_{1}$ and $\mu_{2}$ are obtained by solving the characteristic equation:

$$
\operatorname{det}\left(\left.\frac{\partial T}{\partial \boldsymbol{x}_{0}}\right|_{\boldsymbol{x}_{0}=\boldsymbol{p}}-\mu I\right)=0,
$$

where $I$ is a $2 \times 2$ identity matrix. When $\forall i, \mu_{i} \neq 0$ and $\left|\mu_{i}\right| \neq 1, \boldsymbol{p}$ is called a hyperbolic fixed point. Assuming that $\Re\left(\mu_{1}\right)<\Re\left(\mu_{2}\right)$, let us classify the stability of a hyperbolic fixed point based on its characteristic multipliers: a fixed point is

- completely stable if $\left|\mu_{1}\right|<1$ and $\left|\mu_{2}\right|<1$; and we label the point as ${ }_{0} D$.

- directly unstable if $0<\mu_{1}<1<\mu_{2}$; and we label the point as ${ }_{1} D$.

- inversely unstable if $\mu_{1}<-1<\mu_{2}<1$; and we label the point as ${ }_{1} I$.

- completely unstable if $\left|\mu_{1}\right|>1$ and $\left|\mu_{2}\right|>1$; and we label the point as ${ }_{2} D$.

A left subscript indicates the number of the unstable dimension for the fixed point. Generally, ${ }_{0} D$ is called $a$ node or sink, ${ }_{1} D$ and ${ }_{1} I$ are called saddles, and ${ }_{2} D$ is called a source. Similarly to the fixed point, we classify the stability of the $\ell$-periodic point with denoting each symbol as ${ }_{0} D^{\ell}$ and so on.

We numerically obtain the fixed point $\boldsymbol{p}$ by applying Newton's method to Eq. (8) or Eq. (9). We also numerically obtain the coefficient matrix of Eq. (10) by using the method mentioned in Ref. [14], which presents the computation method to obtain the Jacobian matrix of the hybrid systems.

By perturbing some parameters, we find the parameter sets where the stability of $\boldsymbol{p}$ changes. This is called a local bifurcation of $\boldsymbol{p}$ [7], and each of the parameter sets is called a local bifurcation set. Local bifurcations occur when one of the multipliers of $\boldsymbol{p}$ exceeds unity by changing a parameter. In the system (1), two local bifurcations are possible; the tangent bifurcation $\left(\mu_{i}=1\right)$ and the perioddoubling bifurcation $\left(\mu_{i}=-1\right)$. We numerically obtain the local bifurcation sets by using the method in Ref. [14].

\subsection{Global bifurcations}

Discussing global bifurcations, we generally consider the stable and unstable manifolds of a hyperbolic fixed point $\boldsymbol{p}$. A stable manifold of $\boldsymbol{p}$, which we also call an $\boldsymbol{\omega}$-branch of $\boldsymbol{p}$, is a manifold composed of the points whose image under $T^{k}$ such that $k \rightarrow \infty$ arrives at $\boldsymbol{p}$. An unstable manifold of $\boldsymbol{p}$, which we 

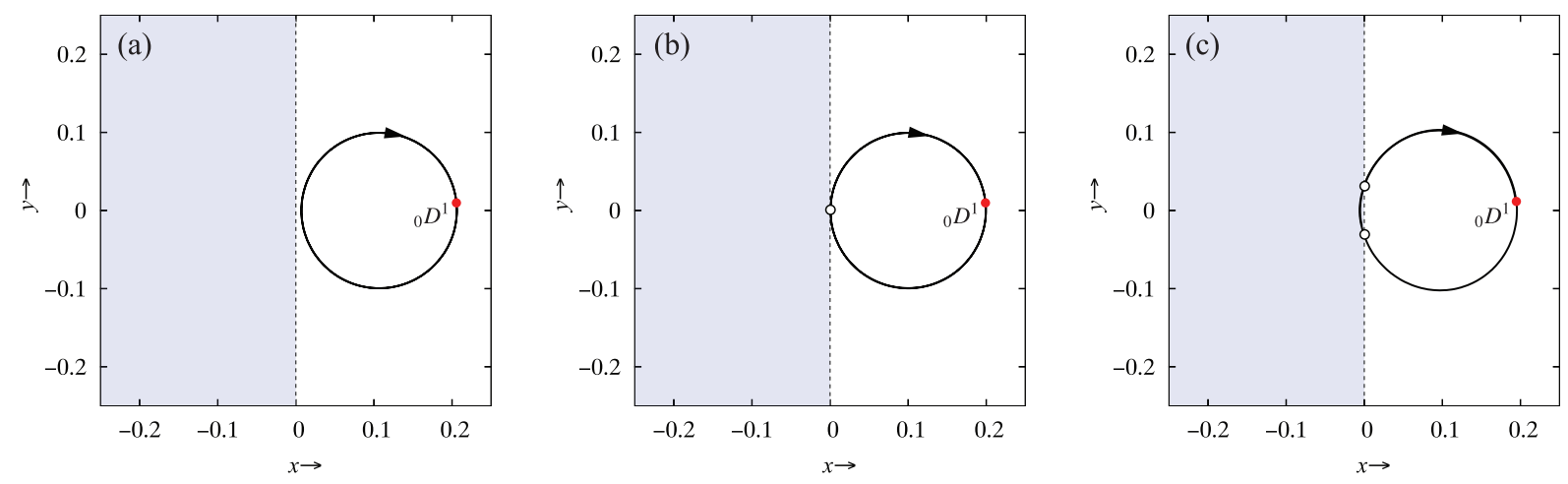

Fig. 2. Phase portraits of the system (1) with $k=0.2, B=0.2, s_{R}=3$ and $s_{L}=1 / 3$. Red points are the Poincare maps of the orbits. (a) Linear periodic orbit with $B_{0}=0.32$. (b) Linear periodic orbit that grazes the border $x=0$ with $B_{0}=0.3$. (c) Nonlinear periodic orbits transversing the border $x=0$ with $B_{0}=0.28$.

also call an $\alpha$-branch of $\boldsymbol{p}$, is a manifold composed of the points whose image under $T^{-k}$ such that $k \rightarrow \infty$ arrives at $\boldsymbol{p}$. They are described as follows:

$$
\begin{aligned}
\omega(\boldsymbol{p}) & =\left\{\boldsymbol{x} \in \boldsymbol{R}^{2} \mid \lim _{k \rightarrow \infty} T^{k}(\boldsymbol{x})=\boldsymbol{p}\right\}, \\
\alpha(\boldsymbol{p}) & =\left\{\boldsymbol{x} \in \boldsymbol{R}^{2} \mid \lim _{k \rightarrow \infty} T^{-k}(\boldsymbol{x})=\boldsymbol{p}\right\} .
\end{aligned}
$$

These two branches determine the behaviors of $T(\boldsymbol{x})$ together with $\boldsymbol{x}$ around $\boldsymbol{p}$. We numerically compute these branches by using the method proposed in Ref. [21]. If an $\alpha$-branch and $\omega$-branch of a fixed point $\boldsymbol{p}$ crosses to each other, the intersections of the branches are called homoclinic points. Especially, in the case that a branch is tangent to the other one at some points, the points of tangency are called degenerate homoclinic or degenerate heteroclinic points. In this study, we consider the homoclinic points observed in the system (1).

Finding a homoclinic point is a required process to identify a horseshoe map which deeply relates to the existence of chaotic saddles [17] (see next section). In addition, obtaining degenerate homoclinic points is recognition of a chaotic region in the parameter space. The appearance of degenerate homoclinic points is referred to as a homoclinic bifurcation, and the parameter set where a homoclinic bifurcation arises is called a homoclinic bifurcation set.

We numerically compute both a homoclinic bifurcation set and corresponding degenerate homoclinic points by the method developed by Yoshinaga et al. in Ref. [16]. The derivatives of $T$ with respect to initial values and parameters are necessary for obtaining the bifurcation set and are numerically calculated by the method proposed in our previous study [14].

The system may have another global bifurcation called a grazing bifurcation. Let us consider the case that a periodic orbit passes near the border within $M_{+}$, as shown in Fig. 2(a). Perturbing a parameter, the periodic orbit might approach the border and finally graze it, as shown in Fig. 2(b). After that, the periodic orbit cannot keep its topological structure anymore and completely disappears. This disappearance, or an appearance inversely, is called a grazing bifurcation of the periodic orbit [22], and the parameter set where grazing bifurcation phenomenon arises is called a grazing bifurcation set. After grazing bifurcation arises, there might remain a periodic orbit whose shape is similar to the disappeared orbit, as shown in Fig. 2(c). In other words, such remaining orbit has exactly different stability from the disappeared orbit.

The condition of grazing bifurcation is that a periodic orbit and the border of the system are tangent to each other at the time $\tau$ :

$$
\left\{\begin{array}{l}
q(\boldsymbol{x}(\tau))=0, \\
\left.\frac{d q}{d t}\right|_{t=\tau}=0 .
\end{array}\right.
$$

We compute grazing bifurcation sets by simultaneously solving Eq. (8) and (14) with Newton's 
method.

\section{Results of analysis}

In this section, we fix some parameters and apply the method mentioned above to the system (1). We explore all periodic orbits of the system (1) in the space $x \in[-5,5]$ and $y \in[-5,5]$ of the state space $\boldsymbol{R}^{2}$. We indicate the existence of horseshoe map required to cause chaos, compare the bifurcation structures of the Duffing equation and the system (1), and finally specify the bending angle to bring bifurcations and chaos.

\subsection{Existence of the horseshoe map}

As a special case, the system (1) becomes the Hamiltonian system if $k=0$. At that time, there are some saddles on the $x$-axis; and the $\alpha$ - and $\omega$-branch of such saddles are symmetric to the $x$-axis. This helps us to understand the horseshoe map from the standpoint of geometry. A horseshoe map is a kind of chaotic map introduced by Smale [17]. If a map is the horseshoe map in some bounded region of the phase space, we know the properties as follows:

- The region has countably many periodic points of the map with arbitrarily high period and all of these periodic points are saddle type (called chaotic saddles).

- The region has uncountably many non-periodic points of the map.

- The region has dense orbits.

Letting $k$ be zero, and observe $\alpha$ and $\omega$ branches of the saddle $\boldsymbol{p}$ as shown in Fig. 3(a). Many transversal homoclinic points are recognized. For this figure, let us choose an orange-colored area $\mathrm{ABCD}$ as shown in Fig. 3(b). The image of ABCD moves along the $\alpha$ branch as $T$ iterates, and it is stretched and folded. After appropriate times of the iteration for $T$, we obtain a purple-colored image A'B'C'D' overlapped on ABCD. In this situation, a horseshoe map is composed. That is, the corresponding symbolic dynamics described by infinite sequences and a shift map imply the existence of chaotic saddles near $\boldsymbol{p}$ [17]. Although the chaotic saddles are not observable for both positive and negative time evolution, at least we can deduce that above transversal homoclinic points and the horseshoe map are initiated by a homoclinic bifurcation where a parameter set gives tangential intersections of $\alpha$ and $\omega$ branches.
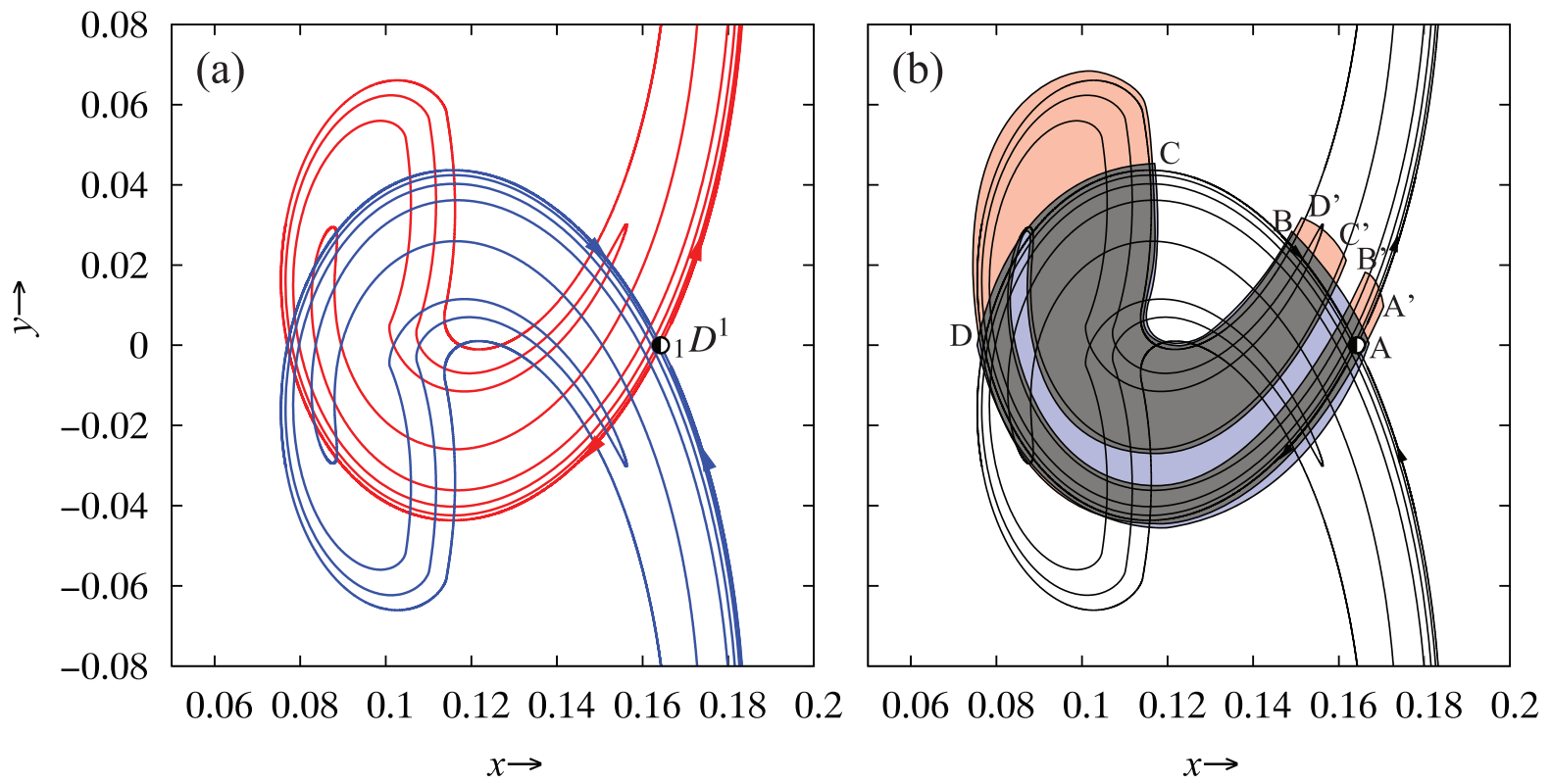

Fig. 3. (a) $\alpha$-branch (red) and $\omega$-branch (blue) of a saddle $D_{1}^{1}$ with $k=$ $0, B_{0}=0.13, B=0.1, s_{L}=1 / 50$, and $s_{R}=3$. (b) A region ABCD (orangecolored) and its image A'B' $\mathrm{C}^{\prime} \mathrm{D}$ ' (purple-colored) under $T$ with the parameters of Fig. 3(a). 
If we increase $k$ or change other parameter values gradually, the horseshoe structure is collapsed, however, it is expected that the complex behavior around the saddle may be preserved or survived near the homoclinic bifurcation since the horseshoe map is structurally stable. Even though the relationship between chaotic attractors and chaotic saddles is not clarified in detail, a chaotic attractor appears via an inverse process of a crisis [23]. We will revisit this homoclinic bifurcation later in this section.

\subsection{Comparison with the Duffing equation}

Let us fix the parameters as $k=0.2, s_{L}=1 / 3$ and $s_{R}=3$, and then we can see bifurcation sets of the system (1) in $B-B_{0}$ plane, as shown in Fig. 4(a). In the following bifurcation diagrams, $G^{\ell}$ is the tangent bifurcation set, $I^{\ell}$ is the period-doubling bifurcation set, $Z^{\ell}$ is the grazing bifurcation set, and $H$ is the homoclinic bifurcation set of a saddle ${ }_{1} D$. The right subscript of each symbol is an identifier of each bifurcation set and right superscript is the period of the periodic orbit.

In the gray shaded region, the system (1) presents the linear periodic orbit, as shown in Fig. 2(a). On the right side of the line $Z_{0}^{1}$, the periodic orbit crosses the border $x=0$ and is not "linear" anymore, as shown in Figs. 2(b) and (c). There exist the period-doubling bifurcation sets $I_{0}^{1}$ and the tangent bifurcation sets $G_{0}^{1}$ and $G_{1}^{1}$ since the periodic orbit is no longer "linear". On the other hands, the system (1) shows two stable periodic orbits with the parameters between $G_{0}^{1}$ and $G_{1}^{1}$. At this time, these two orbits have different amplitudes, as shown in Figs. 5(a) and (c). We call the outer closed curve that has larger amplitude a resonant orbit and the other inner closed curve that has smaller amplitude a non-resonant orbit. There also exists another unstable periodic orbit between them though it is invisible since it is unstable. These three periodic orbits demonstrate a nonlinear resonance phenomenon [24].

Let us compare Fig. 4(a) with Fig. 4(b) which is the bifurcation diagram of the Duffing equation [18]. As a common feature, there are two bifurcation sets $G_{0}^{1}$ and $G_{1}^{1}$ both having an increasing slope. The nonlinear resonances are confirmed in both systems, as shown in Figs. 5(a) and (c), in the region enclosed by $G_{0}^{1}$ and $G_{1}^{1}$. The systems have almost the same structures of the vector field, though the amplitudes of the resonant orbits are quite different. On the other hand, as a different characteristic, there are no similar bifurcation sets except for $G_{0}^{1}$ and $G_{1}^{1}$. In Fig. 4(a), there exist period-doubling bifurcations near $G^{0}$, and there are no other bifurcation sets in the parameter region. Conversely, in Fig. 4(b), there is no period-doubling bifurcation set near $G_{0}^{1}$, and there exist other tangent bifurcation sets $G_{2}^{1}, G_{3}^{1}$ and $G_{4}^{1}$, and some period-doubling bifurcations $I_{1}^{1}$ and $I_{2}^{1}$. In addition, in Fig. 4(a), there is no parameter set showing chaos. In the case of the Duffing equation, there exist the parameter sets showing chaos, e.g., the region enclosed by $I_{1}^{1}$.
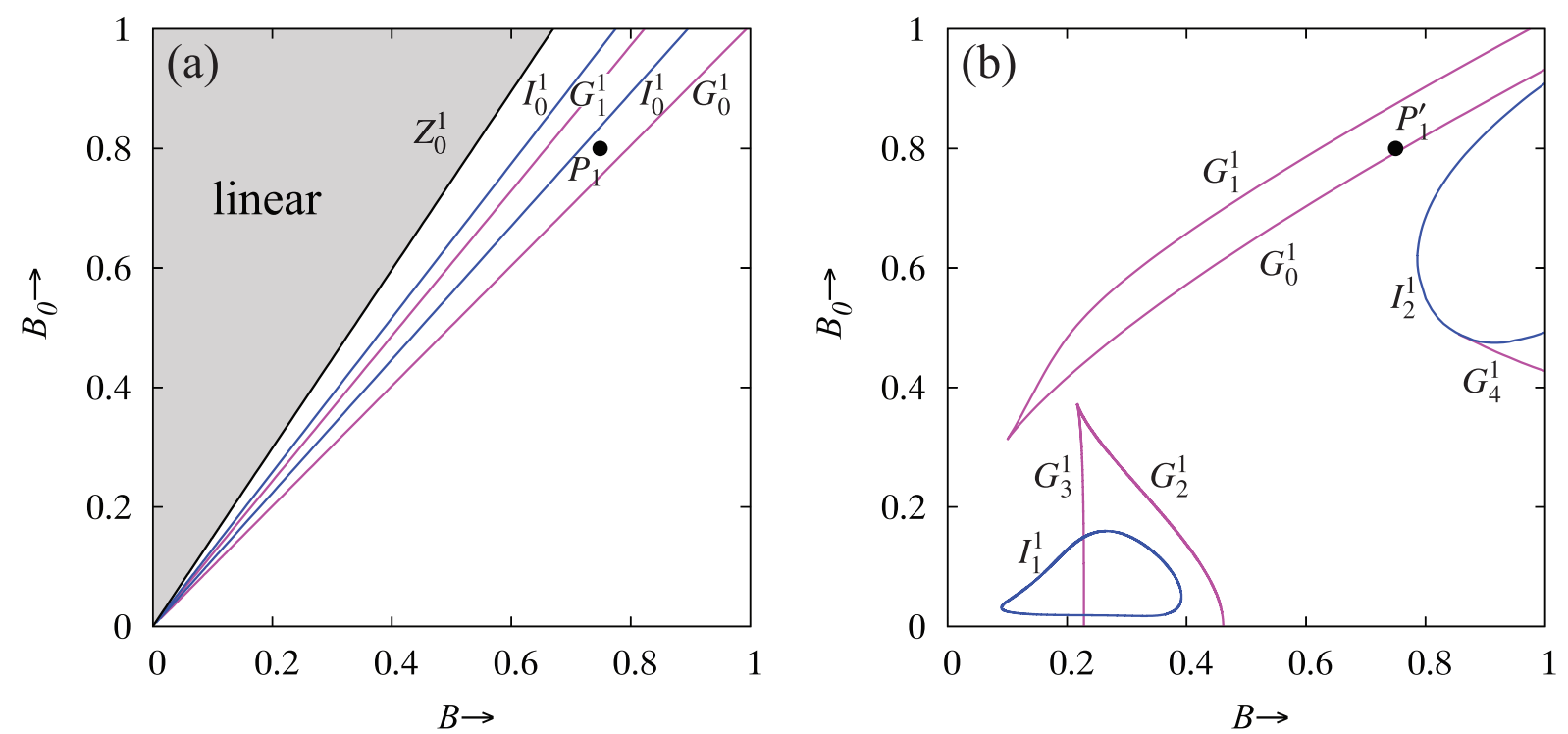

Fig. 4. Bifurcation diagrams of (a) the system (1) and (b) the Duffing equation with $f(x)=x^{3}$ in $B-B_{0}$ plane: $B \in[0,1]$ and $B_{0} \in[0,1]$. 

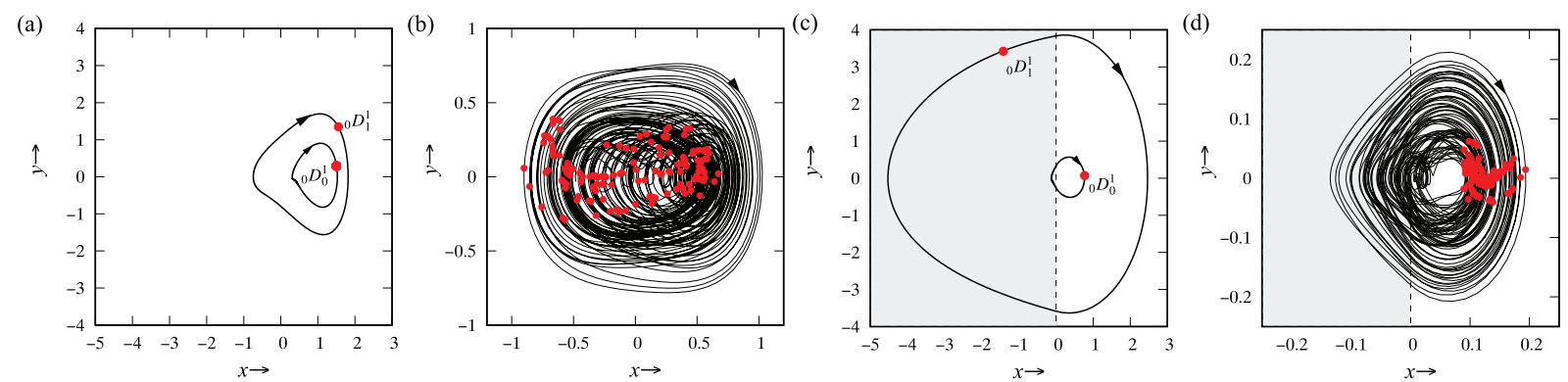

Fig. 5. Phase portraits. (a) and (b) are the orbits of the Duffing equation with $f(x)=x^{3}$, and (c) and (d) are the orbits of the system (1). Red points are the Poincare maps of the orbits. (a) and (c) are resonant and non-resonant orbits and are calculated with $k=0.2, B_{0}=0.8$, and $B=0.75$, which corresponds to $P_{1}$ in Fig. 4(a) and $P_{1}^{\prime}$ in Fig. 4(b). (b) Chaos with $k=0.04$, $B_{0}=0.06$, and $B=0.15$. (d) Chaos with $k=0.04, B_{0}=0.13$, and $B=0.11$.

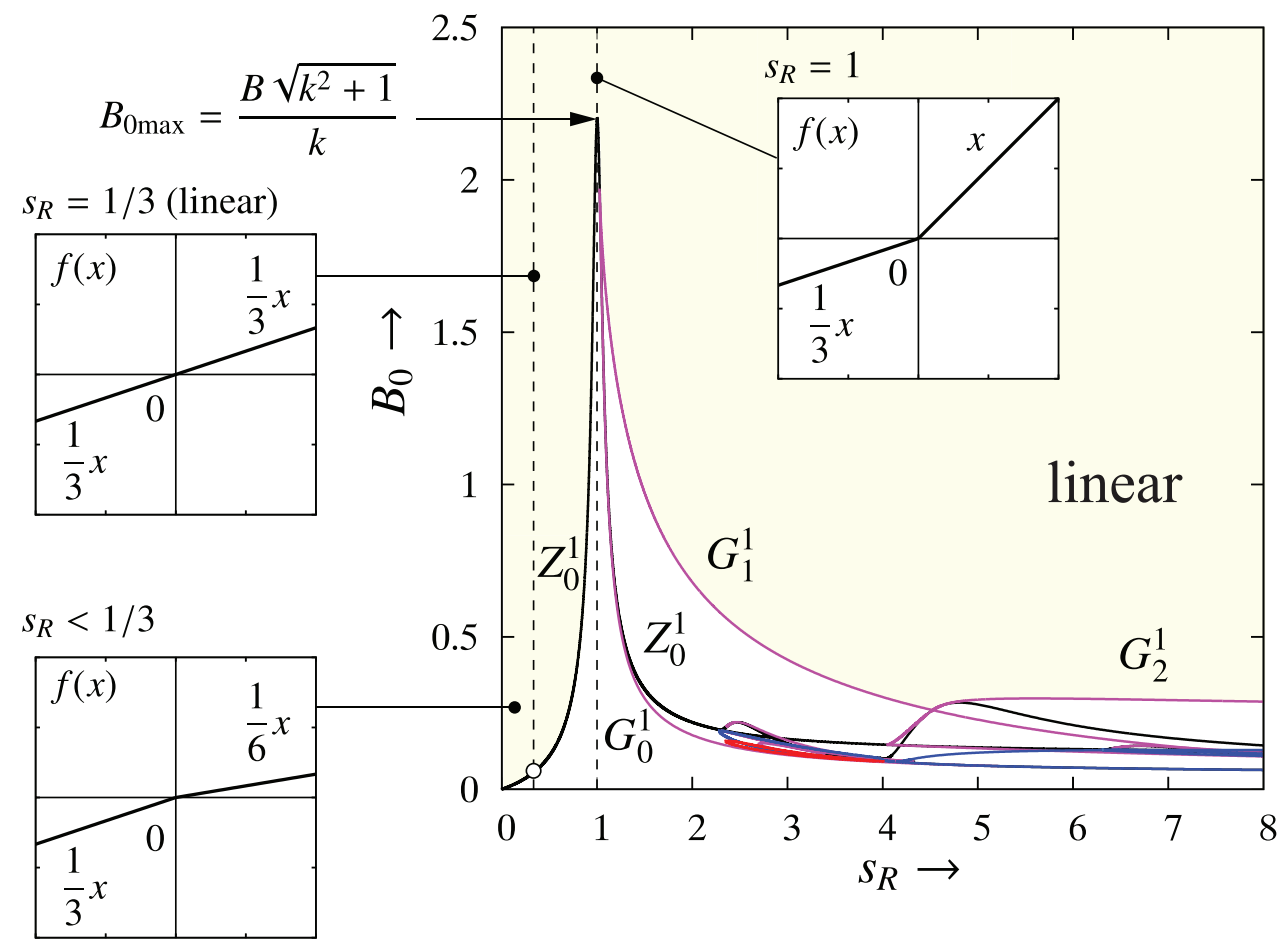

Fig. 6. 2-dimensional bifurcation diagrams of the system (1) in $s_{R}-B_{0}$ plane: $s_{R} \in[0,8]$ and $B_{0} \in[0,2.5]$ with $k=0.05, B=0.11$ and $s_{L}=1 / 3$. Small windows show the shapes of $f(x)$ with each parameter $\left(s_{R}=1 / 6,1 / 3,1\right)$. Vertical lines indicate $s_{R}=1 / 3$, which is the parameter making $f(x)$ linear.

\subsection{A contribution of a broken line to bring bifurcations and chaos}

In this section, we fix the parameters as $k=0.05, B=0.11$, and $s_{L}=1 / 3$, control $B_{0}$ and $s_{R}$ as variable parameters, and finally, consider the relationship between bifurcations and the slope of $f(x)$.

The parameter set $Z_{0}^{1}$ in Fig. 6 is the grazing bifurcation set of a linear periodic orbit; $G_{1}^{1}$ and $G_{2}^{1}$ are tangent bifurcation sets of a periodic point. These curves, $Z_{0}^{1}, G_{1}^{1}$, and $G_{1}^{1}$, form a boundary between linear and nonlinear regions. In the yellow region above these curves, we have linear periodic orbits.

In the parameter region enclosed by $G_{0}^{1}$ and $G_{1}^{1}$, there exists the nonlinear resonance, as shown in Fig. 7(b). Consequently, the system (1) has resonant and non-resonant orbits for the same parameter value. In this system, there arise extremely rich bifurcation phenomena near the non-resonant orbit, as shown in Figs. 7(c) and (d). From now, let us set $B_{0}=0.13$ and confirm these phenomena. The non-resonant orbit firstly appears with the parameters at $G_{0}^{1}$, as shown in Fig. 8(a). Increasing the value of $s_{R}$ from this parameter, period-doubling bifurcation occurs with the parameters at $I_{0}^{1}$ and 
(a)

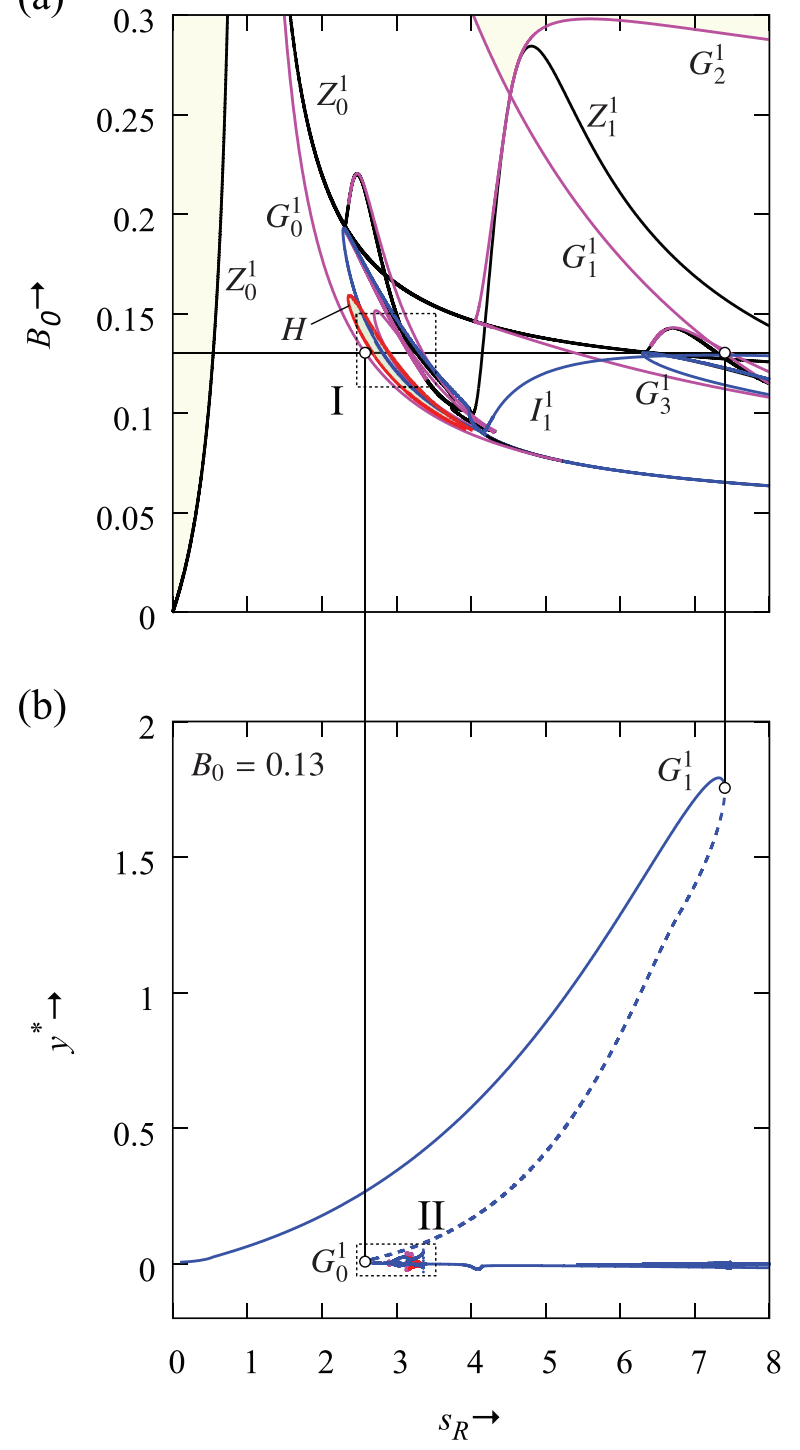

(c)

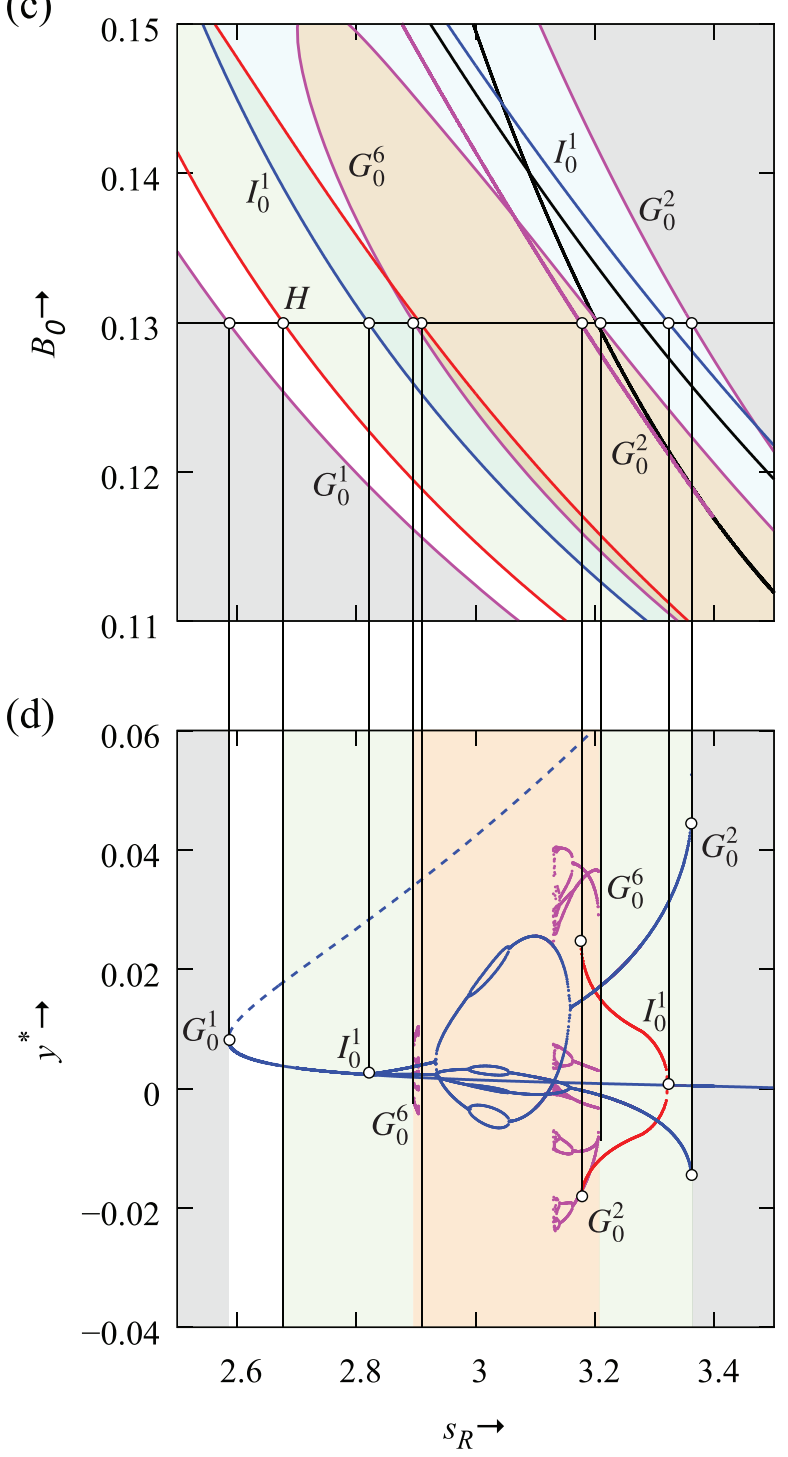

Fig. 7. (a) 2-dimensional bifurcation diagrams of the system (1) in $s_{R}-B_{0}$ plane: $s_{R} \in[0,8]$ and $B_{0} \in[0,0.4]$ with $k=0.05, B=0.11$ and $s_{L}=1 / 3$. (b) 1-dimensional bifurcation diagram of the system (1) with $k=0.05, B_{0}=0.13$, $B=0.11, s_{L}=1 / 3$, and $s_{R} \in[0,8] . y^{*}$ is the coordinate value of $y$ of the Poincaré map (7). Vertical line in (a) indicate $s_{R}=1 / 3$, which is the parameter making $f(x)$ linear, and horizontal line in $(\mathrm{a})$ indicates $B_{0}=0.13$. Solid curves in (b) are attractors, and broken curves are unstable periodic points. (c) Enlargement figures of the box I in (a) and (d) the box II in (b) with $s_{R} \in[2.5,3.5]$.

a 2-periodic orbit immediately arises, as shown in Fig. 8(b). At the same time, the non-resonant orbit gets unstable. Further increasing the value of $s_{R}$, we observe the period-doubling cascade. For simplicity, we have not plotted the bifurcation set of the period-doubling cascade in Fig. 7. For $B_{0}=0.13$, due to the period-doubling cascade, the period of the attractor doubles one after another until there arises an 8-periodic orbit, as shown in Figs. 8(c) and (d). After that, with increasing $s_{R}$ again, the attractor takes the invert scenario of the doubling and its period gets half one after another until there arises a 2-periodic orbit, as shown in Figs. 8(e) and (f). Blue solid curves in Fig. 7(d) show these processes. Notice that the period of the 2-periodic orbit shown in Fig. 8(f) does not get half in these processes and finally disappears by tangent bifurcation with $G_{0}^{2}$. On the other hand, with increasing $s_{R}$, the unstabilized non-resonant orbit gets stable due to period-doubling bifurcation with $I_{0}^{1}$ on the right side, as shown in Fig. 8(g). Conversely, decreasing $s_{R}$ from near $I_{0}^{1}$ on the right side, a 2-periodic orbit arises by the period-doubling bifurcation with $I_{0}^{1}$, as shown in Fig. 8(h). Further 

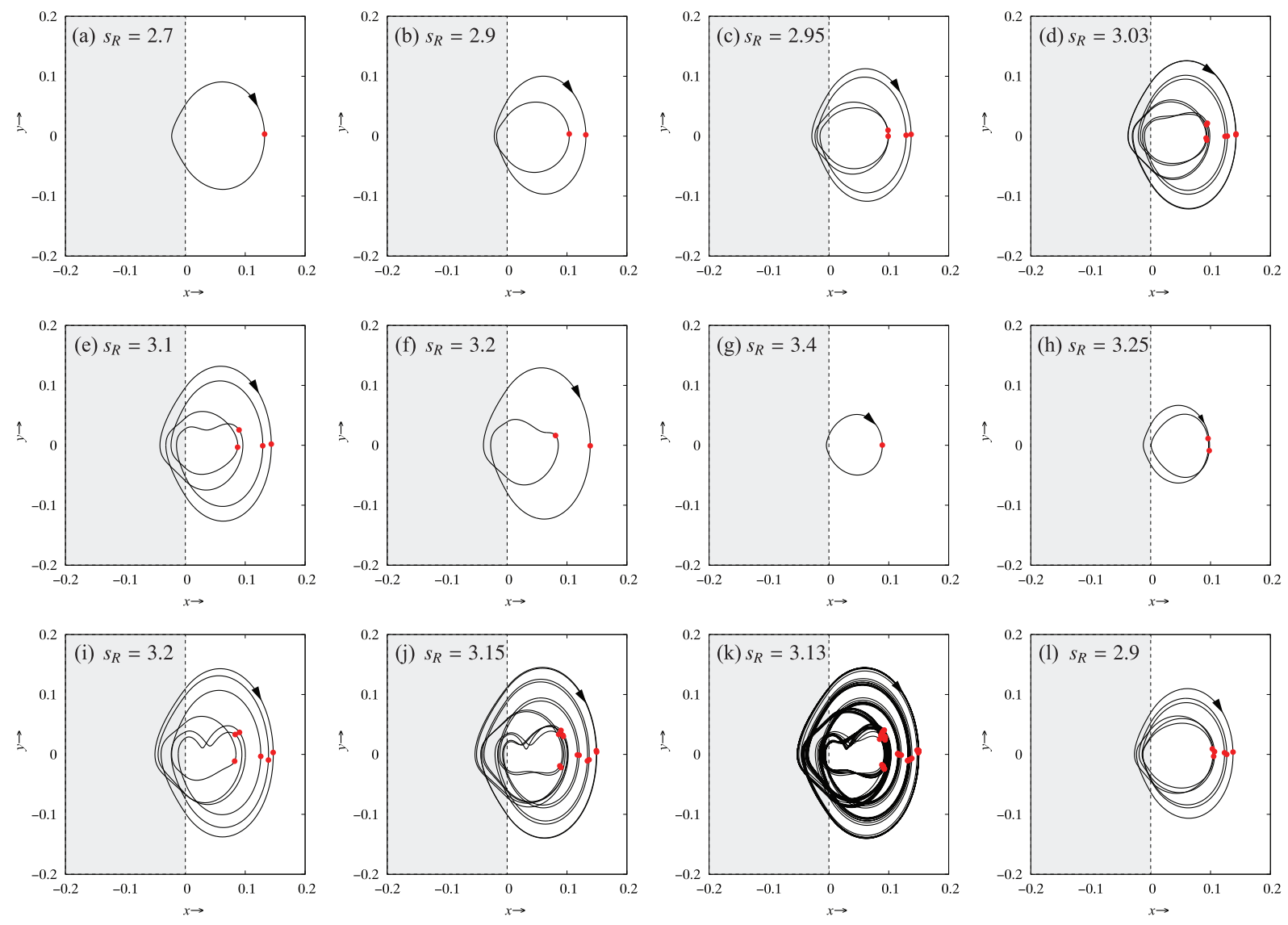

Fig. 8. Phase portraits of the system (1) with $k=0.05, B_{0}=0.13, B=0.11$, $s_{L}=1 / 3$ and each $s_{R}$. Red points are the Poincaré maps of each periodic orbit.

decreasing $s_{R}$, this orbit disappears at $G_{0}^{2}$ on the left side because of the tangent bifurcation.

As the bifurcation which is not concerned with the non-resonant orbit, there arises the tangent bifurcation raising a 6-periodic orbit at $G_{0}^{6}$, as shown in Fig. 8(i). Decreasing $s_{R}$ from near $G_{0}^{6}$ on the right side, period-doubling bifurcation occurs, and a 12-periodic orbit emerges at the same time, as shown in Fig. 8(j). After that, with decreasing $s_{R}$ again, the attractor becomes chaotic, as shown in Fig. 8(k). Further decreasing $s_{R}$, we find a value in the parameter that the attractor suddenly disappears. Besides, the same tangent bifurcation occurs at $G_{0}^{6}$ on the left side, and a 6-periodic orbit appears, as shown in Fig. 8(1); and, as $s_{R}$ increases, it also suddenly disappears. We consider this disappearance is caused by the change of the global property of the state space, i.e., the global bifurcation phenomenon. Let us consider this problem in our future work because it is not the main topic of this paper.

Even though it is not confirmed in the 1-dimensional bifurcation diagram in Fig. 7(d), there also arises a global bifurcation phenomenon for a saddle between the resonant and the non-resonant orbits. Homoclinic bifurcation of the saddle occurs at $H$. Figure 9 shows the stable and unstable manifolds of the saddle with the parameters near $H$.

Setting the values in the shaded region in Fig. 6 as the parameters of the system (1), there not arise bifurcation phenomena. Therefore, the system (1) with parameters in this region shows only one linear periodic orbit but never shows another periodic orbit. Conversely, local and global bifurcation phenomena occur only in the region $s_{R}>k^{2}+1$. In this sense, we consider that $f(x)$ with $s_{R}=k^{2}+1$ includes the minimum bending angle needed to cause the bifurcation phenomena. Thus, the angle is given by

$$
\tan ^{-1}\left(k^{2}+1\right)-\tan ^{-1} s_{L}=0.464896[\mathrm{rad}] .
$$

On the other hand, chaos occurs if there appear homoclinic points in the state space (even if the chaos is attractive or not). From Fig. 7 and Fig. 9, we find homoclinic points when we choose the 
(a)

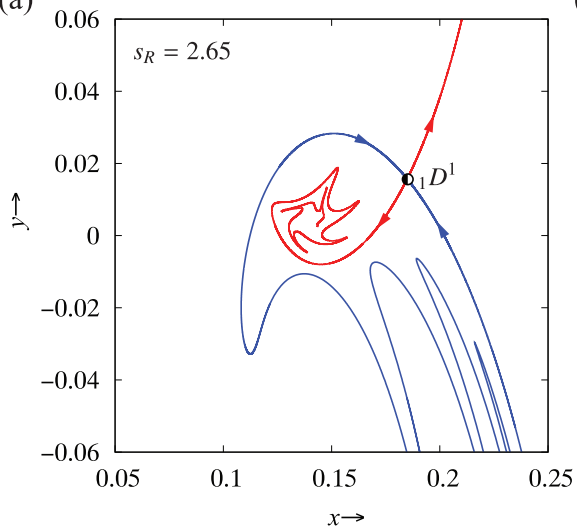

(b)

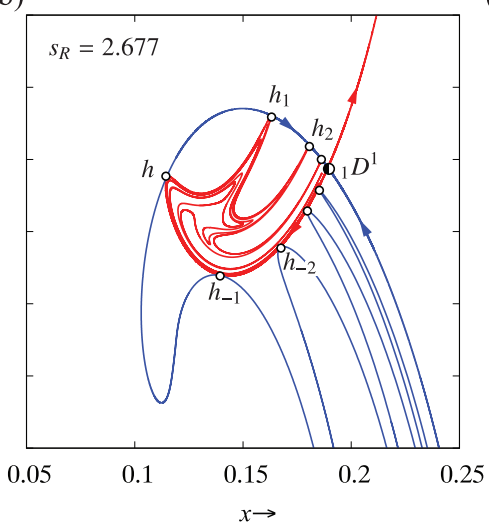

(c)

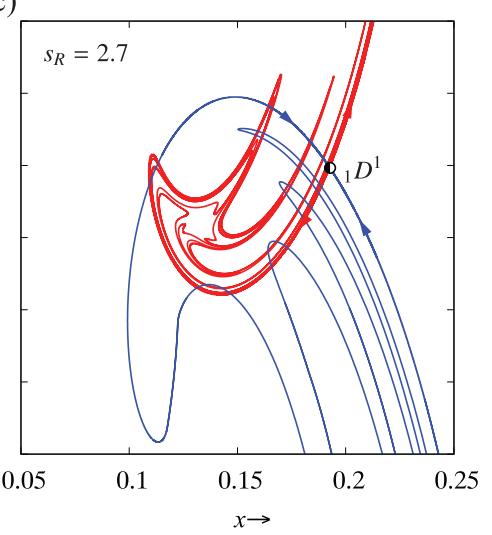

Fig. 9. $\alpha$-branch (red) and $\omega$-branch (blue) of a saddle $D_{1}^{1}$ with $k=0.05$, $B_{0}=0.13, B=0.11, s_{L}=1 / 3$, and each $s_{R}$ : (a) $s_{R}=2.65$, (b) $s_{R}=2.667$, and (c) $s_{R}=2.7$. Outlined circles: $h_{-2}, h_{-1}, h, h_{1}, h_{2}$, are the degenerate homoclinic points of $D_{1}^{1}$.

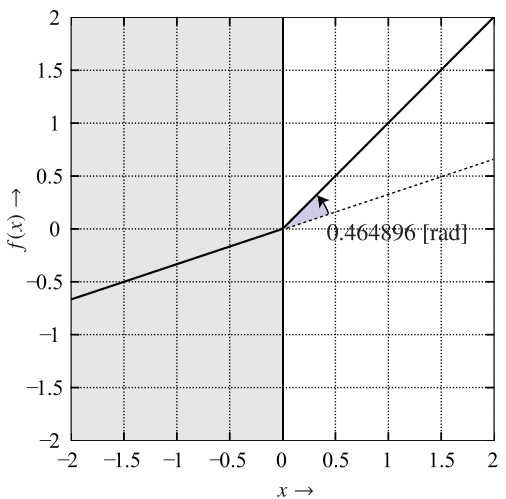

(a) $f(x)$ with $s_{R}=k^{2}+1$.

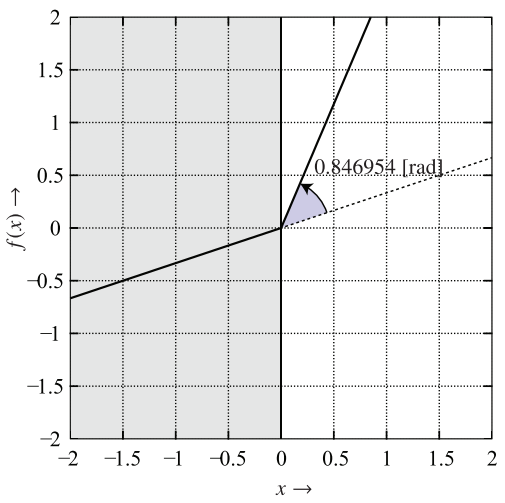

(b) $f(x)$ with $s_{R}=2.3515$.

Fig. 10. Broken lines with the bending angles to cause bifurcations and chaos.

parameters in the space enclosed by $H$ and never homoclinic bifurcations found in the parameter space $s_{R}<2.3515$. Therefore, we consider that $f(x)$ with $s_{R}=2.3515$ has the minimum bending angle needed to cause chaos. The angle is

$$
\tan ^{-1} 2.3515-\tan ^{-1} s_{L}=0.846954[\mathrm{rad}] .
$$

Figure 10 shows $f(x)$ with each $s_{R}$.

\section{Conclusion}

We investigated the minimum bending angle of a broken line bringing bifurcations and chaos to a forced planar system. We proposed a simple dynamical system including a PWL function and analytically introduced the linear periodic orbit with its condition of existence. We confirmed the general definitions for the local stability, local and global bifurcations, and grazing bifurcation and Main results of the analysis of the system (1) are listed below:

- Local, global and grazing bifurcation sets are exactly computed in 2-dimensional bifurcation diagrams.

- The system (1) has almost different bifurcation structures from the Duffing equation.

- Nonlinear resonances arise, that is, three different periodic orbits exist with the same parameter.

- Some periodic orbits caused by tangent bifurcation and period-doubling bifurcation appear near the non-resonant orbit. 
- Visible chaotic attractor occurs from the period-doubling bifurcation of a 6-periodic orbit.

- A horseshoe map emerges in invisible chaos with a particular parameter.

- A grazing bifurcation and tangent bifurcations form boundaries between linear and nonlinear responses.

- The PWL function constructed from the parameter $s_{R}=k^{2}+1$ shows the minimum bending angle to exhibit bifurcation phenomena under $s_{L}=1 / 3$.

- The PWL function constructed from the parameter $s_{R}=2.3515$ shows the minimum bending angle to exhibit chaos under $s_{L}=1 / 3$ and $k=0.05$.

Our next scope is the reason why $s_{R}=k^{2}+1$ is the trigger of exhibiting bifurcations.

\section{References}

[1] E.N. Lorenz, "Deterministic nonperiodic flow," Journal of the Atmospheric Sciences, vol. 20, no. 2, pp. 130-141, 1963.

[2] B. Poddar, F. Moon, and S. Mukherjee, "Chaotic motion of an elastic-plastic beam," Journal of Applied Mechanics, vol. 55, no. 1, pp. 185-189, 1988.

[3] T. Matsumoto, L. Chua, and M. Komuro, "The double scroll," IEEE Trans. on Circuits and Systs., vol. 32, no. 8, pp. 797-818, 1985.

[4] J.C. Sprott, "A new class of chaotic circuit," Physics Letters A, vol. 266, no. 1, pp. 19-23, 2000.

[5] E.M. Izhikevich, "Simple model of spiking neurons," IEEE Trans. Neural Networks, vol. 14, pp. 1569-1572, November 2003.

[6] D. Pierson and F. Moss, "Detecting periodic unstable points in noisy chaotic and limit cycle attractors with applications to biology," Physical review letters, vol. 75, no. 11, p. 2124, 1995.

[7] Y.A. Kuznetsov, Elements of applied bifurcation theory, vol. 112. Springer Science \& Business Media, 2013.

[8] Y. Ma, H. Kawakami, and C.K. Tse, "Bifurcation analysis of switched dynamical systems with periodically moving borders," IEEE Trans. on Circuits and Systs. I, vol. 51, no. 6, pp. 11841193, 2004.

[9] S. Tanaka, T. Matsumoto, and L. Chua, "Bifurcation scenario in a driven RL-diode circuit," Physica D: Nonlinear Phenomena, vol. 28, no. 3, pp. 317-344, 1987.

[10] T. Saito and K. Mitsubori, "Control of chaos from a piecewise linear hysteresis circuit," IEEE Trans. on Circuits and Systs. I, vol. 42, no. 3, pp. 168-172, 1995.

[11] N. Inaba and S. Mori, "Chaos via torus breakdown in a piecewise-linear forced van der Pol oscillator with a diode," IEEE Trans. on Circuits and Systs., vol. 38, no. 4, pp. 398-409, 1991.

[12] T. Kousaka, T. Ueta, and H. Kawakami, "Bifurcation of switched nonlinear dynamical systems," IEEE Trans. Circuits Systs., vol. 46, no. 7, pp. 878-885, 1999.

[13] S. Banerjee, "Coexisting attractors, chaotic saddles, and fractal basins in a power electronic circuit," IEEE Trans. Circuits Systs., vol. 44, no. 9, pp. 847-849, 1997.

[14] Y. Miino, D. Ito, and T. Ueta, "A computation method for non-autonomous systems with discontinuous characteristics," Chaos, Solitons 83 Fractals, vol. 77, pp. 277-285, 2015.

[15] S.W. Shaw and P. Holmes, "A periodically forced piecewise linear oscillator," Journal of sound and vibration, vol. 90, no. 1, pp. 129-155, 1983.

[16] T. Yoshinaga, H. Kitajima, H. Kawakami, and C. Mira, "A method to calculate homoclinic points of a two-dimensional noninvertible map," IEICE Trans. Fundamentals, vol. E80-A, no. 9, pp. 1560-1566, 1997.

[17] S. Smale, "Differentiable dynamical systems," Bulletin of the American mathematical Society, vol. 73 , no. 6 , pp. $747-817,1967$.

[18] H. Kawakami, "Bifurcation of periodic responses in forced dynamic nonlinear circuits: Computation of bifurcation values of the system parameters," IEEE Trans. Circuits and Systs., vol. 31, pp. 248-260, March 1984. 
[19] Y. Zhao, J. Feng, and K.T. Chi, "Stability analysis of periodic orbits of nonautonomous piecewise-linear systems by mapping approach," IEEE Transactions on Circuits and Systems II: Express Briefs, vol. 56, no. 11, pp. 845-849, 2009.

[20] R. Devaney, An introduction to chaotic dynamical systems, Westview Press, 2008.

[21] Z. You, E.J. Kostelich, and J.A. Yorke, "Calculating stable and unstable manifolds," International Journal of Bifurcation and Chaos, vol. 1, no. 03, pp. 605-623, 1991.

[22] P.T. Piiroinen, L.N. Virgin, and A.R. Champneys, "Chaos and period-adding; experimental and numerical verification of the grazing bifurcation," Journal of Nonlinear Science, vol. 14, no. 4, pp. 383-404, 2004.

[23] C. Grebogi, E. Ott, and J.A. Yorke, "Crises, sudden changes in chaotic attractors, and transient chaos," Physica D: Nonlinear Phenomena, vol. 7, no. 1-3, pp. 181-200, 1983.

[24] S. Rajasekar and M.A. Sanjuan, Nonlinear resonances, Springer, 2016. 